\title{
DIRETRIZES GERAIS PARA UMA POLÍTICA NACIONAL DE INCLUSÃO DAS AÇÕES DE DIAGNÓSTICO NO PROCESSO DE ATENÇÃO À SAÚDE: A ATENÇÃO EM ANÁLISES CLÍNICAS E TOXICOLÓGICAS COMO UM DOS EIXOS ESTRUTURANTES
}

\author{
Norberto Rech ${ }^{1}$ \\ Ronald Ferreira dos Santos ${ }^{2}$ \\ Paulo Roberto Boff ${ }^{3}$ \\ Ivan Walter Tierling ${ }^{4}$ \\ Marco Koerich ${ }^{5}$ \\ Anna Paula de Borba Batschauer ${ }^{6}$
}

RECH, N.; SANTOS, R. F. dos; BOFF, P. R.; TIERLING, I. W.; KOERICH, M.; BATSCHAUER, A. P. de B. Diretrizes gerais para uma politica nacional de inclusão das ações de diagnóstico no processo de atenção à saúde: a atenção em análises clínicas e toxicológicas como um dos eixos estruturantes. Arq. Ciênc. Saúde Unipar, Umuarama, 19, n. 3, p. 229-234, set./dez. 2015.

\begin{abstract}
RESUMO: As políticas públicas voltadas para atenção à saúde dependem da participação e envolvimento político, exigem o aprimoramento científico e técnico, além de ferramentas adequadas para a gestão, incorporação de tecnologias e a aplicação de estratégias para a superação das fragmentações dos serviços, na perspectiva concreta das efetivas universalidade e integralidade das ações de saúde, com equidade. $\mathrm{O}$ objetivo deste documento foi propor diretrizes de uma política voltada às análises clínicas e toxicológicas alinhada com as atuais iniciativas do Ministério da Saúde. Foi utilizada como metodologia a análise das discussões e demandas extraídas dos fóruns das Comissões de Análises Clínicas dos Conselhos de Farmácia do Sul do Brasil, tendo como pressupostos as questões conceituais e estratégicas elencadas no presente documento. O resultado deste documento gerou de um conjunto de diretrizes gerais e seis eixos estratégicos, quais sejam, os conceitos fundamentais, o acesso e segurança do paciente, o financiamento e estruturação da rede, a incorporação de tecnologias, o reconhecimento do trabalho na atenção em análises clínicas e a regulação dos serviços. Espera-se que estes eixos possam colaborar com as iniciativas em andamento no Ministério da Saúde, especialmente no tocante à estruturação da rede de diagnóstico e suas interfaces com todas as ações de atenção e cuidado em saúde, incluindo aqueles de caráter complementar às ações desenvolvidas no contexto dos serviços públicos de atenção.
\end{abstract}

PALAVRAS-CHAVE: Análises Clínicas; Diretrizes; SUS.

\section{GENERAL GUIDELINES FOR A NATIONAL POLICY FOR THE INCLUSION OF DIAGNOSIS ACTIONS IN THE PROCESS OF HEALTH CARE: ATTENTION IN CLINICAL AND TOXICOLOGICAL ANALYSIS AS ONE OF THE STRUCTURING AXES}

\begin{abstract}
Public policies for health care depend on the participation and political involvement, requiring scientific and technical improvement, as well as appropriate tools for management, incorporating technologies and implementation strategies to overcome the fragmentation of services with a concrete view of the actual universality and comprehensiveness of health actions with equity. The purpose of this study is to propose guidelines for a policy aimed at clinical and toxicological analysis in line with the current Ministry of Health initiatives. It used the analysis of discussions and demands extracted from the forums of Clinical Analysis Committees of South Pharmacy Councils Brazil as its methodology, taking the conceptual and strategic issues listed in this document as assumptions. The result of this document has generated a set of general guidelines and six strategic axes, namely, the fundamental concepts, patient access and safety, network financing and structuring, technology incorporation, work recognition in clinical analysis care, and service regulation. It is expected that these axes can collaborate with ongoing initiatives at the Ministry of Health, especially regarding the structure of the diagnostic network and its interfaces with all care and health care actions, including those of complementary character to the actions developed in the context of public health care services.

KEYWORDS: Clinical analysis; Guidelines; H.U.S.
\end{abstract}

\section{Indrodução}

O desafio de construir cotidianamente o Sistema Único de Saúde (SUS), além da participação e envolvimento político na formulação das políticas públicas que impactam o campo da saúde, exige o aprimoramento científico e técnico, a adoção de ferramentas adequadas para a gestão, a incorporação crítica de diferentes tecnologias e a aplicação de estratégias eficientes e dinâmicas para a superação das fragmentações dos serviços, na perspectiva concreta das efe- tivas universalidade e integralidade das ações de saúde, com equidade.

Nesse contexto, mesmo com todos os avanços na construção do SUS no Brasil, o setor saúde permanece, em diferentes aspectos e intensidades, vivenciando a crise na forma de se produzir em saúde identificada no início dos anos 1.990 , com indução ao modelo produtor de procedimentos, caracterizado como "modelo médico hegemônico" (CECÍLIO, 1994; MERHY, CECÍLIO, NOGUEIRA, 1992; DONANGELO, FERREIRA, 1976).

DOI: https://doi.org/10.25110/arqsaude.v19i3.2015.5553

${ }^{1}$ Farmacêutico. Mestre em Ciências Farmaceuticas. Docente do Departamento de Ciências Farmacêuticas da Universidade Federal de Santa Catarina (UFSC) Florianópolis - SC, Brasil.

${ }^{2}$ Farmacêutico. Mestre em Ciências Farmaceuticas. Presidente da Federação Nacional dos Farmacêuticos (FENAFAR), São Paulo - SP, Brasil.

${ }^{3}$ Farmacêutico. Mestre em Ciências Farmaceuticas . Diretor da Agencia de Inovação e Empreendedorismo da Universidade do Sul de Santa Catarina (UNISUL) - Florianópolis - SC, Brasil.

${ }^{4}$ Farmacêutico. Responsável Técnico e Coordenador do laboratório Municipal de Análises Clínicas e Ambientais. Chapecó - SC, Brasil.

${ }^{5}$ Farmaêutico. Especialista em Microbiologia e Doenças infecto contagiosas. Proprietátio do laboratório Nossa Senhora de Fátima . Florianópolis - SC, Brasil. ${ }^{6}$ Farmacêutica. Doutora em Análises Clínicas e Toxicológicas. Docente da Universidade do Vale do Itajaí (UNIVALI), Itajaí - SC, Brasil. 
A superação das fragmentações de serviços, de programas, de ações e de práticas clínicas requer que o modelo de atenção à saúde considere as alterações dinâmicas no perfil epidemiológico da população, possibilitando a necessária e constante adequação da organização da atenção e da gestão do SUS, tendo como um dos fundamentos o estabelecimento sistemático da coerência entre a oferta de serviços e as necessidades de atenção à saúde (MENDES, 2011; BRASIL, 2010a). Em tal modelo, a assistência à saúde é centrada no ato prescritivo que produz o procedimento, não sendo consideradas as determinações do processo saúde-doença centradas nos determinantes sociais ambientais e relacionadas às subjetivações, singularizantes, valorizando apenas as dimensões biológicas (MALTA, MERHY, 2010).

\section{Comentários}

Este formato do modelo de atenção produz custos elevados e crescentes, pois utiliza, como insumos principais, os recursos tecnológicos centrados em exames e medicamentos (tecnologias duras), como se esses tivessem um fim em si mesmo e fossem capazes de restabelecer a saúde por si só. São produzidos atos desconexos sem uma intervenção articulada e cuidadora, reduzindo-se a eficácia da assistência prestada (MALTA et al., 2004).

Como uma das formas de superação das incoerências entre oferta de serviços e as necessidades em saúde, a formação de redes integradas e regionalizadas de atenção à saúde tem se mostrado como forma de organização de sistemas de saúde eficaz para responder a alguns desses desafios estruturais e epidemiológicos, trazendo melhores resultados para os indicadores de saúde (BRASIL, 2012; OPAS, OMS, 2008; MENDES, 2008).

Avançar na qualificação da atenção e da gestão em saúde requer forte decisão dos gestores do SUS, enquanto protagonistas do processo instituidor e organizador do sistema de saúde. Uma das principais iniciativas que direcionaram as ações do Ministério da Saúde nesse sentido foi a publicação da Portaria no 4.279, de 30 de dezembro de 2010, que estabelece diretrizes para a estruturação da Rede de Atenção à Saúde (RAS) como estratégia para superar a fragmentação da atenção e da gestão nas regiões de saúde e aperfeiçoar o funcionamento político-institucional do SUS, com vistas a assegurar ao usuário o conjunto de ações e serviços que necessita com efetividade e eficiência (BRASIL, 2012).

As RAS constituem-se em arranjos organizativos formados por ações e serviços de saúde, com diferentes configurações tecnológicas e missões assistenciais, articulados de forma complementar e com base territorial, e têm diversos atributos, entre eles, destaca-se: a atenção básica estruturada como primeiro ponto de atenção e principal porta de entrada do sistema, constituída de equipe multidisciplinar que cobre toda a população, integrando, coordenando o cuidado e atendendo às suas necessidades de saúde (BRASIL, 2011).

No que tange à organização da RAS, torna-se, portanto, essencial a definição quanto ao modelo de atenção à saúde a ser adotado, considerando-se seu conceito de

sistema lógico que organiza o funcionamento da RAS, articulando, de forma singular, as relações entre a população e suas subpopulações estratificadas por riscos, os focos das intervenções do sistema de atenção à saúde e os diferentes tipos de intervenções sanitárias, definido em função da visão prevalecente da saúde, das situações demográficas e epidemiológicas e dos determinantes sociais da saúde, vigentes em determinado tempo e em determinada sociedade (BRASIL, 2012; BRASIL, 2010).

A implantação da RAS exige uma intervenção concomitante sobre as doenças agudas e crônicas, em uma organização que construa a intersetorialidade para a promoção da saúde, contemple a integralidade dos saberes com o fortalecimento do apoio matricial, considere as vulnerabilidades de grupos ou populações e suas necessidades, qualificando e fortalecendo as ações de atenção (BRASIL, 2010).

No processo de estruturação da Rede de Atenção à Saúde, Atenção Básica à Saúde (ABS) tem caráter estratégico relevante, especialmente por caracterizar-se como o ponto de atenção com maior capilaridade e potencial para identificar as necessidades de saúde da população e realizar a estratificação de riscos que subsidiará a organização do cuidado em toda a rede. É responsável também por realizar ações de promoção e de proteção da saúde, prevenção de agravos, diagnóstico, tratamento, reabilitação, redução de danos e manutenção da saúde para a maior parte da população (BRASIL, 2011).

Nesse sentido, além da expansão e permanente qualificação da ABS, o seu papel de ordenamento da rede de atenção e na coordenação do cuidado exige ações que possibilitem o desenvolvimento do cuidado integral à saúde, com impactos na situação de saúde, na autonomia das pessoas e nos determinantes e condicionantes de saúde das coletividades, observando-se a necessária articulação com os demais pontos de atenção da rede (BRASIL, 2012; BRASIL, 2011).

No que se refere à qualificação da ABS, é essencial a consideração de que suas ações devem responder de forma efetiva às necessidades de saúde da população adstrita, as quais exigem permanentes adequações da infraestrutura dos serviços, da oferta diagnóstica e terapêutica, bem como aos processos de trabalhos respectivos.

Todavia, para que a ABS seja realmente resolutiva, em especial, no cuidado às pessoas com doenças crônicas, é fundamental que a RAS disponha de fortes sistemas de apoio diagnóstico e terapêutico, sendo que os sistemas e ferramentas de informação, tais como o uso do prontuário eletrônico, fornecem subsídios precisos para a estratificação de risco da população, planejamento e acompanhamento do cuidado na RAS, favorecendo também a comunicação entre a ABS e os diferentes pontos de atenção. (BRASIL, 2012).

Estes aspectos são importantes, particularmente na medida em que os fluxos e condições para encaminhamentos devem ser definidos no contexto da construção das linhas de cuidado. Mesmo enquanto persistir o acompanhamento na atenção especializada, a ABS deve continuar informada da situação de saúde do usuário, bem como acompanhar o desenvolvimento do plano de cuidado. Os critérios para a alta devem ser pactuados, bem como as condições para o seguimento desse usuário na ABS (BRASIL, 2012).

Assim, necessário se torna reconhecer o papel estruturante da adoção do conceito de "linha de cuidado", enquanto escopo teórico do qual devem derivar entendimentos e práticas que permeiem não apenas a gestão do sistema de 
saúde, mas principalmente as práticas e os fazeres no processo de atenção à saúde, consideradas como ações necessariamente multiprofissionais.

A Linha do cuidado é a imagem pensada para expressar os fluxos assistenciais seguros e garantidos ao usuário, no sentido de atender às suas necessidades de saúde. É como se ela desenhasse o itinerário que o usuário faz por dentro de uma rede de saúde, incluindo segmentos não necessariamente inseridos no sistema de saúde, mas que participam de alguma forma da rede, tal como entidades comunitárias e de assistência social (FRANCO, MAGALHÃES Jr., 2003).

Entretanto, é essencial referir que a Linha do Cuidado (LC) é diferente dos processos de referência e contra referência, apesar de incluí-los também. Ela difere, pois, não funciona apenas por protocolos estabelecidos, mas também pelo reconhecimento de que os gestores dos serviços podem pactuar fluxos, reorganizando o processo de trabalho, a fim de facilitar o acesso do usuário às Unidades e Serviços aos quais necessita (FRANCO, MAGALHÃES Jr., 2003).

No sentido mais amplo, a concepção da LC parte da missão institucional do estabelecimento/serviço de saúde, provendo mecanismos que garantam o cuidado (CECÍLIO, 1997). A LC é alimentada por recursos/insumos que expressam as tecnologias a serem consumidas pelos usuários durante o processo de assistência ao beneficiário, funcionando de forma sistêmica e operando vários serviços. Esta tem início na entrada do usuário em qualquer ponto do sistema que opere a assistência: seja no atendimento domiciliar, na equipe de saúde da família/atenção básica, em serviços de urgência, nos consultórios, em qualquer ponto onde haja interação entre o usuário e o profissional de saúde. A partir deste lugar de entrada, abre-se um percurso que se estende, conforme as necessidades do beneficiário, por serviços de apoio diagnóstico e terapêutico, especialidades, atenção hospitalar e outros (MALTA et al., 2004; MERHY, CECÍLIO, 2003).

Nesse aspecto, a inclusão das ações de diagnóstico como parte do processo de atenção à saúde, no contexto da Linha do Cuidado e considerando a organização do sistema em rede, traduzida na estruturação de Rede de Atenção à Saúde (RAS), exige não apenas a incorporação crítica e sustentável de tecnologias, mas demanda a reidentificação dos profissionais atuantes no âmbito das análises clínicas e toxicológicas, entre os quais se destacam os profissionais farmacêuticos, na perspectiva da ação multiprofissional voltada ao acolhimento, à atenção integral e ao acompanhamento dos usuários do SUS no seu processo de promoção da saúde, de prevenção da doença e de recuperação da saúde, considerados os seus diferentes condicionantes.

Entretanto, a inclusão acima referida exige a adequada contextualização da oferta desses serviços no país.

Os dados apresentados pelo DATA-SUS, referentes a 2012 , indicam que cerca de $80 \%$ da população brasileira é atendida exclusivamente pelo SUS, sendo que apenas cerca de $20 \%$ têm cobertura por planos privados de atenção à saúde. As regiões Norte e Nordeste apresentam percentual de cobertura de cerca de $90 \%$ pelo SUS, enquanto que as regiões Sudeste e Sul apresentam um percentual de cobertura pública de atenção à saúde da ordem de $62 \%$ e $77 \%$, respectivamente.

A maior participação do setor privado na oferta e cobertura na atenção à saúde tende a gerar indução à crescen- te concentração de serviços e oferta de tecnologias em saúde, com destaque para a área do diagnóstico.

Os dados de cobertura por planos de saúde ou pelo sistema público de atenção à saúde devem ser correlacionados com a origem da oferta dos serviços, uma vez que a oferta pública conta, também, com a participação da rede complementar privada, incluindo aí a rede de diagnóstico.

Segundo dados da Agência Nacional de Saúde Suplementar (ANS), em 2009, existiam 16.657 laboratórios de Análises Clínicas no Brasil, sendo que 4.917 qualificados como públicos e 11.240 privados. Desse total, 5.468 laboratórios eram privados e integravam a rede complementar do SUS. Dessa forma, a cobertura diagnóstica total vinculada ao SUS é de $62,35 \%$, o que totaliza 10.385 Laboratórios de Análises Clínicas.

Quanto aos laboratórios de Anatomia Patológica/ Citologia compreendem 5.854 Laboratórios, destes 1.300 laboratórios públicos, 4.554 privados, sendo 1.973 laboratórios privados e integrantes de rede complementar do SUS. Assim, o serviço de Anatomia Patológica e Citopatologia tem uma cobertura de 57,89\%, totalizando 3.273 laboratórios prestam serviços ao SUS.

Importante ressaltar que o modelo atual de financiamento nas Análises Clínicas revela-se fragmentado desde a década de 80 , com a instituição da Tabela SUS, este modelo está desarticulado da organização atual dos serviços em saúde. $\mathrm{O}$ atual modelo não apresenta contribuição para o desenvolvimento tecnológico nacional, o qual se observa uma dependência internacional em equipamentos e insumos neste setor.

O número médio de consultas por habitante em todo o território nacional é de 2,67, gerando 1,21 procedimentos diagnósticos por consulta (DATA-SUS 2012). Entretanto, segundo Tribunal de Contas da União, esses números apresentaram crescente aumento entre 2002 até 2010 (3,3 e 4,04 respectivamente) em consultas médicas por habitantes/ano. Segundos esses últimos dados, e considerando as informações do DATA-SUS 2012, a cobertura de ações de diagnóstico seria da ordem de 2,48 procedimentos/habitante/ano, realizada pela rede própria e pela rede complementar do SUS. Este dado gera impactos na definição do financiamento e, principalmente, na forma de organização dos serviços.

Considerando a estratégia das redes de Atenção, a ampliação do percentual de cobertura pela rede própria e complementar, bem como a definição de políticas de indução à incorporação de novas tecnologias, representam estratégias fundamentais para a oferta dos serviços diagnósticos no contexto de Atenção a Saúde, nos seus diferentes níveis de complexidade.

A ampliação da rede de assistência ao diagnóstico pública e complementar pode assumir o papel estratégico norteador da definição dos critérios para a incorporação de tecnologias, para a definição de custos e garantia da qualidade dos serviços diagnósticos, sendo que esta ultima perspectiva apresenta inequívocas relações com a política governamental de segurança do paciente e com a política de financiamento.

Considerando as Linhas de Cuidado como estratégia de organização da Atenção à Saúde, incluindo a estruturação das Redes de Atenção, a ampliação da capilaridade dos serviços de assistência ao diagnóstico representa papel importante na ampliação do acesso e atendimento das de- 
mandas geradas no sistema de saúde, proporcionando a integralidade do cuidado.

No que se refere ao financiamento, aponta-se a necessidade de se construir uma nova forma de remuneração dos procedimentos diagnósticos, especialmente na perspectiva da integralidade das Linhas de Cuidado e do consequente impacto na ampliação da agregação de tecnologias e cobertura no acesso da população às ações de diagnóstico, considerando-se os diferentes níveis de complexidade da atenção à saúde.

A ampliação da rede de prestação de serviços de apoio diagnóstico deve considerar uma parcela significativa (38\%) de prestadores de serviços laboratoriais da rede privada não vinculados ao SUS. Nesse aspecto, a Política Nacional de Saúde, a qual inclui o conjunto das diretrizes de garantia de acesso qualificado das pessoas ás ações de saúde, inclusive aquelas de apoio ao diagnóstico representado pelo segmento das Análises Clínicas, certamente gera impactos no número de empregos diretos e indiretos no setor.

Portanto, identificar-se menos com as tecnologias e mais com o processo de cuidado centrado nas pessoas que demandam as ações de saúde, é passo essencial para uma nova inserção sistêmica dos profissionais das Análises Clínicas e dos seus fazeres no mundo do trabalho em saúde e, em particular, do seu fazer no contexto do Sistema Único de Saúde, também consideradas as suas interfaces com a rede complementar de atenção.

Tendo como pressupostos as questões conceituais e estratégicas elencadas no presente documento, entendem os Conselhos Regionais de Farmácia dos estados de Santa Catarina, Rio Grande do Sul e Paraná ser necessária à apresentação de um conjunto de diretrizes gerais e eixos estratégicos que possam colaborar com as iniciativas em andamento no Ministério da Saúde, especialmente no tocante à estruturação da rede de diagnóstico e suas interfaces com todas as ações de atenção e cuidado em saúde, incluindo aqueles de caráter complementar às ações desenvolvidas no contexto dos serviços públicos de atenção.

Assim, os Conselhos Regionais de Farmácia dos estados de Santa Catarina, Rio Grande do Sul e Paraná apresentam as seguintes sugestões, as quais certamente serão acrescidas daquelas colaborações decorrentes do intenso e contínuo processo de debates e formulações ocorridos durante as três edições do Fórum Sul Brasileiro de Análises Clínicas, os quais reuniram profissionais farmacêuticos atuantes no âmbito das Análises Clínicas nos estados da Região Sul do Brasil

\section{PROPOSTA}

\section{DIRETRIZES GERAIS PARA UMA POLÍTICA NACIONAL DE INCLUSÃO DAS AÇÕES DE DIAGNÓSTICO NO PROCESSO DE ATENÇÃO À SAÚDE: A ATENÇÃO EM ANÁLISES CLÍNICAS E TOXICOLÓGICAS COMO UM DOS EIXOS ESTRUTURANTES}

\section{Eixo Estratégico 1: Conceitos Fundamentais:}

1. A Política Nacional de Inclusão das Ações de Diagnóstico no Processo de Atenção à Saúde deve ser conside- rada como política pública norteadora para a formulação de políticas setoriais, entre as quais se destacam a política de ciência, tecnologia e inovação em saúde, de desenvolvimento industrial e de formação de recursos humanos, dentre outras, garantindo a intersetorialidade inerente ao sistema de saúde do país.

2. As ações no âmbito das Análises Clínicas devem ser consideradas como parte estratégica integrante da Politica Nacional de Inclusão das Ações de Diagnóstico no Processo de Atenção à Saúde devendo estar articuladas com os princípios e eixos estratégicos da Política Nacional de Assistência Farmacêutica, especialmente no que tange as ações de saúde voltadas às linhas de cuidado.

\section{Eixo Estratégico 2: Acesso e segurança do paciente:}

\section{Diretrizes gerais:}

a. Ampliação do acesso dos usuários ao processo de atenção à saúde por parte dos profissionais atuantes no contexto das ações de diagnóstico e da atenção em análises clínicas, superando os limitantes identificados entre a incorporação e uso de tecnologias e seu distanciamento das atividades relacionadas ao cuidado em saúde.

b. Ampliação da capacidade de atendimento da demanda real pelos serviços e ações de diagnóstico no contexto do cuidado em saúde, nos seus diferentes níveis de atenção e em atendimento às necessidades reais em saúde.

c. Ampliação e consolidação das articulações entre as ações do cuidado em saúde, incluindo aquelas relativas ao diagnóstico e ao tratamento, com garantia da integralidade das ações de saúde e envolvendo tanto a rede pública com a rede privada complementar.

d. Conformação da rede de diagnóstico em Análises Clínicas com a participação pública e privada, no processo de atenção à saúde, articulada com as regiões de saúde de modo a garantir a equidade regional na oferta de serviços de diagnóstico, respeitando os demais princípios e diretrizes do SUS.

Eixo Estratégico 3: Financiamento e estruturação da rede, incluindo a rede complementar:

\section{Diretrizes gerais:}

a. Construção de redes de acesso, incluindo os municípios de referência para as redes regionais de atenção.

b. Otimização dos recursos no contexto das redes de atenção, com estabelecimento de instrumentos de TI para o acompanhamento do paciente e sua relação com os serviços de saúde (locais, regionais e de âmbito nacional), associado ao processo de estruturação das redes regionais e loco-regionais.

c. Utilização da capacidade instalada da rede privada complementar no processo de atenção à saúde envolvendo o diagnóstico e a atenção em análises clínicas, com estímulo ao adensamento tecnológico no setor e sua utilização em benefício dos usuários do Sistema Único de Saúde. 
d. A superação do modelo fragmentado de financiamento, adotando a garantia da integralidade do cuidado, como forma inovadora de remuneração dos serviços de apoio diagnóstico.

i. Elaborar protocolos clínicos para patologias que ainda não foram contempladas nas linhas de cuidado, com a participação do segmento de Análises Clínicas nas etapas prévias às possíveis consultas públicas.

ii. Permanente atualização dos protocolos clínicos já definidos, com a participação do segmento de Análises Clínicas nas etapas prévias às possíveis consultas públicas.

iii. Aplicar os protocolos clínicos para a demanda de exames de apoio ao diagnóstico no contexto das linhas de cuidado.

iv. Definir instrumentos para a efetiva internalização nos serviços daqueles exames previstos nos respectivos protocolos clínicos, considerando-se a avaliação econômico-epidemiológica e seus impactos na resolutividade das ações de saúde e na redução/otimização dos custos operacionais do sistema nacional de saúde.

v. Estruturação dos serviços de apoio laboratorial ao diagnóstico na rede pública de atenção à saúde, considerando os diferentes níveis de complexidade e as realidades loco-regionais.

e. Definição de mecanismos adequados de análise dos custos para a manutenção dos serviços de apoio laboratorial ao diagnóstico, tanto na rede pública quanto na rede privada complementar, considerando:

i. A participação do segmento de Análises Clínicas na construção destes mecanismos.

ii. As etapas Pré-analíticas, Analíticas e Pós-analíticas.

iii. As ações integrantes das etapas anteriores àquelas consideradas Pré-analíticas e que impactam nos custos das tecnologias necessárias aos serviços de apoio laboratorial ao diagnóstico e acompanhamento.

iv. As diferentes complexidades tecnológicas.

v. Os impactos da demanda sobre os custos para a manutenção dos serviços.

vi. A integralidade da atenção à saúde, no contexto das linhas de cuidado.

\section{Eixo Estratégico 4: Incorporação de tecnologias:}

\section{Diretrizes gerais:}

a. Estímulo à definição e uso de instrumentos e ferramentais metodológicos que possibilitem a incorporação crítica de tecnologias e procedimentos que resultem tanto na otimização de custos como na melhoria dos serviços de diagnóstico, seja na rede pública ou na rede privada de atenção complementar, considerando-se os conceitos de RAS e Linhas do Cuidado.

b. Inclusão da rede complementar no processo de implantação de testes rápidos, com agregação dos conhecimentos e práticas já existentes na rede pública e na rede complementar de diagnóstico, associando-se esse processo a internalização e a ampliação das ações de atenção à saúde dos usuários para os quais são demandadas as atividades de diagnóstico.

Eixo Estratégico 5: Reconhecimento do trabalho na atenção em análises clínicas e toxicológicas:

\section{Diretrizes gerais:}

a. Garantir a representação do trabalho dos profissionias de saúde em todos os fóruns/grupos de trabalho/ câmaras que venham definir regras/normas ou outros instrumentos que impactem nas atividades do diagnóstico laboratorial e no processo de atenção em analises clínicas e toxicológicas, enquanto parte do cuidado em saúde.

b. Desenvolvimento da gestão do trabalho considerando o fato de tratar-se de atividade multiprofissional, mas que deposita em algumas categorias maiores responsabilidades, em particular as profissões de Farmacêutico, Médico e Biomédico.

c. Fortalecimento do potencial da área do diagnóstico para desenvolvimento da Educação Permanente em Saúde, que compreende o conjunto de alternativas educacionais, de forma articulada, visando o desenvolvimento de ações voltadas à aprendizagem significativa no e para o processo de trabalho, entendido como eixo para a transformação das práticas, de forma participativa e contextualizada, objetivando a melhoria da qualidade dos serviços de atenção à saúde.

d. Humanização do atendimento, garantindo os princípios do SUS e qualificando as equipes integradas focadas no cuidado ao paciente e garantindo a equidade.

e. Implementação e ampliação de programas de capacitação e educação continuada, em todos os níveis profissionais envolvidos nas linhas de cuidado, tanto na rede pública quanto na rede privada complementar.

\section{Eixo Estratégico 6: Regulação dos serviços:}

\section{Diretrizes gerais:}

a. Adoção de instrumentos e mecanismos articulados de gestão que possibilitem a estruturação e a administração adequadas da rede de laboratórios regionais por parte do município sede do laboratório central, observando-se a regulação sob a tutela do consórcio regional.

b. Adoção de instrumentos e mecanismos articulados que possibilitem os estabelecimento de metas de cobertura do diagnóstico e a previsão financeira através das pactuações no âmbito dos consórcios regionais estabelecidos.

c. Implementação de ações e mecanismos que permitam que a previsão da cobertura passe a ser integrada com a rede dos Laboratórios Estaduais, com repasse de verbas e reagentes do Estado e do Ministério da Saúde, consideradas as respectivas pactuações.

d. Efetiva integração das ações de suporte ao diagnóstico no contexto da rede nacional de diagnóstico, in- 
cluindo a Atenção Básica e a estruturação das Redes de Atenção à Saúde, considerados os pressupostos estruturantes definidos no escopo das Linhas do Cuidado em saúde.

e. A definição de mecanismos e instrumentos adequados para o direcionamento da demanda de ações de apoio laboratorial ao diagnóstico à rede complementar de atenção, considerando as características tecnológicas dos serviços, as características locais e loco-regionais, a oferta e manutenção de ações de saúde dos prestadores dos serviços no contexto das linhas de cuidado, o acesso dos usuários aos serviços qualificados e a resolutividade das ações de saúde, nos seus diferentes níveis de complexidade.

f. A construção de uma Política de Vigilância Sanitária que garanta o acesso aos serviços de diagnóstico laboratorial, seguro, eficaz e de qualidade, bem como estabelecer mecanismos adequados para a regulação e monitoração do mercado de insumos e serviços estratégicos no apoio diagnóstico.

\section{Referências}

BRASIL. Ministério da Saúde. Portaria no 4.279, de 30 de dezembro de 2010. Estabelece diretrizes para organização da Rede de Atenção à Saúde no âmbito do Sistema Único de Saúde (SUS). Brasília-DF, Diário Oficial da União, Seção 1, 31 dez. 2010.

BRASIL. Ministério da Saúde. Portaria no 2.488, de 21 de outubro de 2011. Aprova a Política Nacional de Atenção Básica, estabelecendo a revisão de diretrizes e normas para a organização da atenção básica, para a Estratégia Saúde da Família (ESF) e o Programa de Agentes Comunitários de Saúde (PACS). Brasília-DF, Diário Oficial da União, Seção 1, 22 out. 2011.

BRASIL. Ministério da Saúde. Secretaria de Atenção à Saúde. Departamento de Atenção Básica. Documento de diretrizes para o cuidado das pessoas com doenças crônicas nas Redes de Atenção à Saúde e nas linhas de cuidado prioritárias / Ministério da Saúde. Secretaria de Atenção à Saúde. Departamento de Atenção Básica. - Brasília: Ministério da Saúde, 2012. 34 p.: il. - (Série B. Textos Básicos de Saúde).

CECÍLIO, L.C.O. Uma sistematização e discussão de tecnologia leve de planejamento estratégico aplicada ao setor governamental. In: MERHY, E.E.; ONOKO, R. (Orgs.). Inventando a mudança na saúde. São Paulo: Hucitec, 1994.

CECÍLIO, L.C.O. Uma sistematização e discussão de tecnologia leve de planejamento estratégico aplicada ao setor governamental. In: MERHY, E.E.; ONOKO, R. (Orgs.). Agir em saúde, um desafio para o público. São Paulo: Hucitec, 1997. p.151-67.

DONNANGELO, M.C.F.; PEREIRA, L. Saúde e sociedade. São Paulo: Duas Cidades, 1976.
FRANCO, T.B \& MAGALHÃES Jr., H.. A Integralidade e as Linhas de Cuidado; in Merhy, E.E. et al, O Trabalho em Saúde: Olhando e Experienciando o SUS no Cotidiano. Hucitec, São Paulo, 2003.

MALTA, D.C. et al. Perspectivas da regulação na saúde suplementar diante dos modelos assistenciais. Cienc. Saude Colet., v.9, n.2, p.433-44, 2004.

MALTA, D.C.; MERHY, E.E. The path of the line of care from the perspective of non- transmissible chronic diseases. Interface - Comunic., Saude, Educ., v.14, n.34, p.593-605, jul./set. 2010.

MENDES, E. V. As redes de atenção à saúde. Rev. Med. Minas Gerais, v. 18, p. 3-11, 2008. (Suplemento 4)

MENDES, E. V. As redes de atenção à saúde. Brasília: Organização Pan-Americana da Saúde, 2011.

MERHY, E.E.; CECÍLIO, L.C.O.; NOGUEIRA, R.C. Por um modelo tecno-assistencial da política de saúde em defesa da vida: contribuição para as conferências de saúde. In: CONFERÊNCIA NACIONAL DE SAÚDE, DESCENTRALIZANDO E DEMOCRATIZANDO O CONHECIMENTO, 9., 1992, Brasília. Cadernos... Brasília, 1992. p.91-6. v.1.

MERHY, E.E.; CECÍLIO, L.C.O. A integralidade do cuidado como eixo da gestão hospitalar. Campinas: Unicamp, 2003. (mimeogr.).

\section{ORGANIZAÇÃO PAN-AMERICANA DA SAÚDE} (OPAS); ORGANIZAÇÃO MUNDIAL DA SAÚDE (OMS). La Renovación de la Atención Primaria de Salud en las Américas. Redes Integradas de Servicios de Salud. Conceptos, Opiciones de Política y Hoja de Ruta para su implementación en las Américas. Outubro 2008.

Recebido: 26/05/2015 Aceito: $11 / 12 / 2015$ 